\title{
Particle Filter Based Fault-tolerant ROV Navigation using Hydro-acoustic Position and Doppler Velocity Measurements
}

\author{
Zhao, Bo; Blanke, Mogens; Skjetne, Roger
}

Published in:

9th IFAC International Conference on Manoeuvring and Control of Marine Craft Manoeuvring and Control of Marine Craft

Link to article, DOI:

10.3182/20120919-3-IT-2046.00048

Publication date:

2012

Document Version

Early version, also known as pre-print

Link back to DTU Orbit

Citation (APA):

Zhao, B., Blanke, M., \& Skjetne, R. (2012). Particle Filter Based Fault-tolerant ROV Navigation using Hydroacoustic Position and Doppler Velocity Measurements. In 9th IFAC International Conference on Manoeuvring and Control of Marine Craft Manoeuvring and Control of Marine Craft (pp. 280-286) https://doi.org/10.3182/20120919-3-IT-2046.00048

\section{General rights}

Copyright and moral rights for the publications made accessible in the public portal are retained by the authors and/or other copyright owners and it is a condition of accessing publications that users recognise and abide by the legal requirements associated with these rights.

- Users may download and print one copy of any publication from the public portal for the purpose of private study or research.

- You may not further distribute the material or use it for any profit-making activity or commercial gain

- You may freely distribute the URL identifying the publication in the public portal 


\title{
Fault Tolerant ROV Navigation System Based on Particle Filter using Hydro-acoustic Position and Doppler Velocity Measurements
}

\author{
Bo Zhao* Mogens Blanke ${ }^{* * *}$ Roger Skjetne ${ }^{* * *}$ \\ * Centre for Ships and Ocean Structures, Norwegian University of \\ Science and Technology, 7491 Trondheim, Norway (e-mail: \\ bo.zhao@ntnu.no) \\ ** Automation and Control Group, Department of Electrical \\ Engineering, Technical University of Denmark, 2800 Kgs. Lyngby, \\ Denmark (e-mail: mb@elektro.dtu.dk) \\ *** Department of Marine Technology, Norwegian University of \\ Science and Technology, 7491 Trondheim, Norway (e-mail: \\ roger.skjetne@ntnu.no)
}

\begin{abstract}
This paper presents a fault tolerant navigation system for a remotely operated vehicle (ROV). The navigation system uses hydro-acoustic position reference (HPR) and Doppler velocity $\log$ (DVL) measurements to achieve an integrated navigation. The fault tolerant functionality is based on a modified particle filter. This particle filter is able to run in an asynchronous manner to accommodate the measurement drop out problem, and it overcomes the measurement outliers by switching observation models. Simulations with experimental data show that this fault tolerant navigation system can accurately estimate the ROV kinematic states, even when sensor failures appear frequently.
\end{abstract}

Keywords:

Particle filter; remotely operated vehicle; fault tolerant navigation.

\section{INTRODUCTION}

ROVs are widely used in various subsea operations, typically in offshore hydrocarbon exploration and production. A large amount of these operations are safety critical, and accurate positioning and control of the ROVs are required. In this aspect, it is necessary to realize high-precision and fault tolerant ROV navigation.

Commonly used ROV navigation sensors have been discussed in Kinsey et al. (2006). These sensors use different principles and have different features. Hence, their update rate, precision, and range are generally different. ROVs are usually equipped with several types of sensors to form an integrated navigation system that overcomes the disadvantages of each type of sensor, and to obtain an accurate position estimation. Applications of ROV integrated navigation are reported in Blain et al. (2003), Kinsey and Whitcomb (2004), and Dukan et al. (2011). In Blain et al. (2003), a navigation system which uses Kalman filtering to merge data received from an acoustic position system, a bathymeter, and a DVL has been developed and implemented in simulation and also in a real submarine. A more mature application is given in Kinsey and Whitcomb (2004). The new result in Dukan et al. (2011) show the integrated navigation system of an ROV with an extended Kalman filter.
In practice it is common that we encounter disturbances on the raw measurements due to sensor faults and noise. If disturbed measurements are used in the estimation of the system states, the effect is difficult to predict. Sensor faults can be diagnosed by checking the consistency of the measurements from the redundant sensors. However, because of limitations, it is not always possible to install redundant sensors on the ROVs. In this case, making use of all available information from different types of sensors to achieve a model-based software redundancy is preferred.

In the past decade, particle filters have been widely studied and proved to be powerful tools in nonlinear and/or nonGaussian system state estimation. Yardim et al. (2011) gives a review of using particle filtering techniques in ocean acoustics. An application of a particle filter for ROV navigation can be found in Zhao et al. (2012). It has also been shown that particle filters are suitable to handle the estimation problem on jump Markov systems, which is a reasonable model of systems subject to failures. Some applications of using this technique in fault tolerant sensor systems can be found in Caron et al. (2007) and Tafazoli and Sun (2006).

In this paper we consider designing a fault tolerant navigation system of an ROV with HPR and DVL. As observed in the ROV sea trials, the HPR and DVL suffer especially from the following failures; 


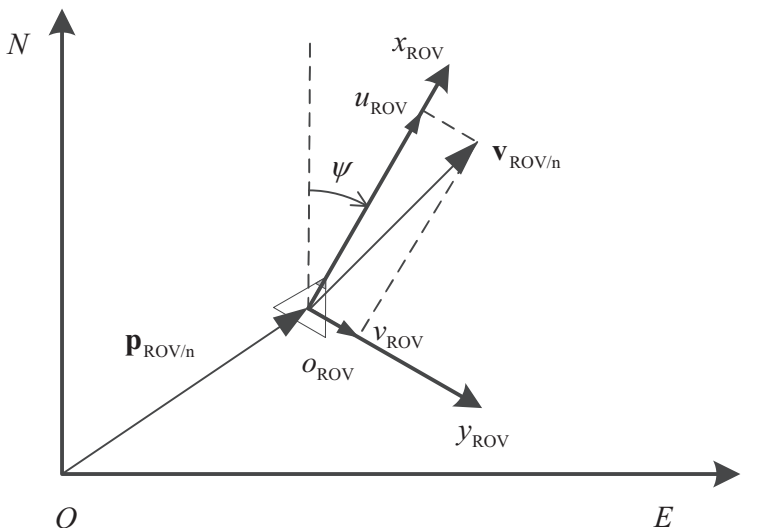

Fig. 1. ROV navigation: body and NE coordinate systems.

(1) HPR drop outs.

(2) HPR measurement outliers.

(3) DVL drop outs.

Besides these three failure modes, another practical issue to be handled is the update rate of the HPR measurements that become uneven when the ROV dives to deep water.

The paper is organized as follows. An ROV kinematic model is given in Section 2, and the HPR and DVL measurement models in fault-free condition are also studied. Based on these fault-free models, a particle filter based navigation algorithm is given in Section 3. Then in Section 4, failure modes of the sensors are listed and studied. Section 5 gives the details of the modifications to the particle filter to handle the listed failure modes. Then a simulation study is given in Section 6 to verify the proposed navigation system.

\section{SYSTEM MODELING}

\subsection{System Description}

The navigation problem considered in this paper is motivated by full-scale ROV experiments conducted by the Applied Underwater Robotics Laboratory (AUR-Lab) of NTNU. The AUR-Lab aims at developing new technology for marine monitoring and ocean observation. Designing a robust and high-performing ROV navigation system is essential to this development.

The operational setup of the ROV experiment is to use a support vessel performing station-keeping or low-speed tracking, while the ROV is controlled from an onboard command station. Figure 1 shows the definitions of the reference frames and corresponding position and velocity vectors. The earth-fixed North-East (NE) frame are indicated with ' $n$ '. A body-fixed frame for the ROV is defined with origin at a given fixed center point $O_{\mathrm{ROV}}$, the $x_{\mathrm{ROV}}-$ axis is along the vessel longitudinal direction, and the $y_{\mathrm{ROV}}$-axis is along the vessel transversal direction. Vectors expressed in the ROV body-fixed frame are indicated by 'ROV'. For instance, the DVL measures the ROV surge velocity $u_{R O V}$ and ROV sway velocity $v_{R O V}$ along the $x_{\mathrm{ROV}}-$ and $y_{\mathrm{ROV}}-$ axis, respectively. And the position and linear velocity vectors of the ROV with respect to the NEframe decomposed in the NE-frame are named as $\mathbf{p}_{\mathrm{ROV} / \mathrm{n}}^{\mathrm{n}}$ and $\mathbf{v}_{\mathrm{ROV} / \mathrm{n}}^{\mathrm{n}}$, respectively.

\subsection{ROV Kinematic Model}

At the present stage, the research is focusing on estimating the ROV kinematic states by only using the motion sensors, meaning that the propulsion of the ROV is not considered. Hence, we aim for a noise driven kinematic model. A discrete kinematic model in the NE frame is

$$
\underbrace{\left[\begin{array}{c}
\mathbf{p}_{\mathrm{ROV} / \mathrm{n}, k+1}^{\mathrm{n}} \\
\mathbf{v}_{\mathrm{ROV} / \mathrm{n}, k+1}^{\mathrm{n}}
\end{array}\right]}_{\mathbf{x}_{k+1}}=\underbrace{\left[\begin{array}{cc}
\mathbf{I} & T \mathbf{I} \\
\mathbf{0} & \mathbf{I}
\end{array}\right]}_{\mathbf{A}_{x}} \underbrace{\left[\begin{array}{c}
\mathbf{p}_{\mathrm{ROV} / \mathrm{n}, k}^{\mathrm{n}} \\
\mathbf{v}_{\mathrm{ROV} / \mathrm{n}, k}^{\mathrm{n}}
\end{array}\right]}_{\mathbf{x}_{k}}+\underbrace{\left[\begin{array}{c}
\frac{1}{2} T^{2} \mathbf{I} \\
T \mathbf{I}
\end{array}\right]}_{\mathbf{B}_{x}} \mathbf{w}_{a, k},
$$

where $\mathbf{w}_{a, k}$ is the white process noise sequence, $k$ denotes the time index, I is 2-by-2 unit matrix, and $T$ is the sampling interval. The model is written compactly as

$$
\mathbf{x}_{k+1}=\mathbf{A}_{x} \mathbf{x}_{k}+\mathbf{B}_{x} \mathbf{w}_{a, k}
$$

where $\mathbf{x}_{k}$ following from (1) is the kinematic state vector of the ROV, and $\mathbf{A}_{x}, \mathbf{B}_{x}$ also follow from (1). Equation (2) can be transformed into an equivalent stochastic process as

$$
p\left(\mathbf{x}_{k+1} \mid \mathbf{x}_{k}\right)=\mathbf{N}\left(\mathbf{A}_{x} \mathbf{x}_{k}, \operatorname{Cov}\left[\mathbf{B}_{x} \mathbf{w}_{a, k}\right]\right),
$$

where $\mathbf{N}(\boldsymbol{\mu}, \boldsymbol{\Sigma})$ denotes the multivariate normal density function with mean $\boldsymbol{\mu}$ and covariance $\boldsymbol{\Sigma}$.

\subsection{Measurement Modeling}

HPR Measurement The HPR system positions an underwater target by measuring its acoustic distance and direction, so the measurement is originally made in spherical coordinates, and then transformed to Cartesian coordinates by the build-in algorithm of HPR system. Hence, there will be correlation between the measurement in each direction of the Cartesian coordinates. The covariance of the HPR measurement noise is estimated recursively from data. With measurement at instant $k$ being $\mathbf{p}_{A, k}$ and $\tilde{\mathbf{p}}_{A}=\mathbf{p}_{A}-\overline{\mathbf{p}}_{A}$, where $\overline{\mathbf{p}}_{A}$ is a moving window average of $\mathbf{p}_{A}$, the estimate of the covariance $\boldsymbol{\Sigma}_{A}$ is

$$
\begin{aligned}
\boldsymbol{\Sigma}_{A, N} & =\frac{1}{N+1} \sum_{k=1}^{N}\left(\tilde{\mathbf{p}}_{A, k}\left(\tilde{\mathbf{p}}_{A, k}\right)^{\top}\right) \\
& =\frac{N}{N+1}\left(\boldsymbol{\Sigma}_{A, N-1}+\frac{1}{N}\left(\tilde{\mathbf{p}}_{A, N}\right)\left(\tilde{\mathbf{p}}_{A, N}\right)^{\top}\right),
\end{aligned}
$$

then, the HPR measurement $\mathbf{p}_{A}$ has the multivariate normal distribution

$$
p\left(\mathbf{p}_{\mathrm{A}, k} \mid \mathbf{p}_{\mathrm{ROV} / \mathrm{n}, k}^{\mathrm{n}}\right)=\mathbf{N}\left(\mathbf{p}_{\mathrm{ROV} / \mathrm{n}, k}^{\mathrm{n}}, \boldsymbol{\Sigma}_{A, k}\right) .
$$

DVL measurement The DVL measures the velocity of the ROV with respect to the NE frame. Decomposed in the ROV body-fixed frame, it is expressed as $\mathbf{v}_{\mathrm{ROV} / \mathrm{n}}^{\mathrm{ROV}}=$ $\left[\begin{array}{ll}u_{\mathrm{ROV}} & v_{\mathrm{ROV}}\end{array}\right]^{\top}$. To transform the DVL measurement into the NE frame where the kinematic equation of the ROV is given, the heading of the ROV $\psi_{\mathrm{ROV}}$ is introduced. The ROV heading is estimated from the measurement of a fluxgate compass and a yaw rate gyro (Dukan et al. (2011)). Then $\mathbf{v}_{\mathrm{ROV} / \mathrm{n}}^{\mathrm{ROV}}$ is related to the general ROV kinematics by

$$
\mathbf{v}_{\mathrm{ROV} / \mathrm{n}}^{\mathrm{ROV}}=\mathbf{R}^{\top}\left(\psi_{\mathrm{ROV}}\right) \mathbf{v}_{\mathrm{ROV} / \mathrm{n}}^{\mathrm{n}},
$$

where $\mathbf{R}\left(\psi_{\mathrm{ROV}}\right)$ is the corresponding $3 \mathrm{DOF}$ rotation matrix. 
Assuming that the DVL measurement noise in each direction are independent identical normally distributed, the DVL velocity measurement $\mathbf{v}_{\mathrm{D}, k}$ can be seen as a multivariate normally distributed random vector with expectation $\mathbf{v}_{\mathrm{ROV} / \mathrm{n}}^{\mathrm{ROV}}$ and covariance matrix $\boldsymbol{\sigma}_{\mathrm{D}}^{2} \mathbf{I}$, where $\boldsymbol{\sigma}_{\mathrm{D}}^{2}$ is the variance of the DVL measurement noise in each direction. To sum up, the DVL measurement $\mathbf{v}_{\mathrm{D}, k}$ can be expressed as

$$
p\left(\mathbf{v}_{\mathrm{D}, k} \mid \mathbf{v}_{\mathrm{ROV} / \mathrm{n}, k}^{\mathrm{n}}\right)=\mathbf{N}\left(\mathbf{R}^{\top}\left(\psi_{\mathrm{ROV}}\right) \mathbf{v}_{\mathrm{ROV} / \mathrm{n}, k}^{\mathrm{n}}, \boldsymbol{\sigma}_{\mathrm{D}}^{2} \mathbf{I}\right) .
$$

\section{PARTICLE FILTER DESIGN}

Collecting the ROV kinematic model (2), and the measurement models (5), (7) yields the following navigation model of the ROV as

$$
\begin{aligned}
p\left(\mathbf{x}_{k+1} \mid \mathbf{x}_{k}\right) & =\mathbf{N}\left(\mathbf{A}_{x} \mathbf{x}_{k}, \operatorname{Cov}\left[\mathbf{B}_{x} \mathbf{w}_{a, k}\right]\right) \\
p\left(\mathbf{p}_{\mathrm{A}, k} \mid \mathbf{p}_{\mathrm{ROV} / \mathrm{n}, k}^{\mathrm{n}}\right) & =\mathbf{N}\left(\mathbf{p}_{\mathrm{ROV} / \mathrm{n}, k}^{\mathrm{n}}, \mathbf{\Sigma}_{A}\right) \\
p\left(\mathbf{v}_{\mathrm{D}, k} \mid \mathbf{v}_{\mathrm{ROV} / \mathrm{n}, k}^{\mathrm{n}}\right) & =\mathbf{N}\left(\mathbf{R}^{\top}\left(\psi_{\mathrm{ROV}}\right) \mathbf{v}_{\mathrm{ROV} / \mathrm{n}, k}^{\mathrm{n}}, \boldsymbol{\sigma}_{\mathrm{D}}^{2} \mathbf{I}\right)
\end{aligned}
$$

where $\mathbf{C}_{\mathrm{p}}=\left[\begin{array}{ll}\mathbf{I}_{2 \times 2} & \mathbf{0}_{2 \times 2}\end{array}\right]$ and $\mathbf{C}_{\mathrm{v}}=\left[\begin{array}{ll}\mathbf{0}_{2 \times 2} & \mathbf{I}_{2 \times 2}\end{array}\right]$.

The particle filter applied in this application follows the sampling importance re-sample algorithm in Arulampalam et al. (2002), which is given in Appendix A.

With this algorithm, the posterior density of the system state at $k$ can be estimated as

$$
p\left(\mathbf{x}_{k} \mid\left[\begin{array}{ll}
\mathbf{p}_{\mathrm{A}, l: k} & \mathbf{v}_{\mathrm{D}, l: k}
\end{array}\right]\right) \approx \sum_{i=1}^{N_{s}} w_{k}^{i} \delta\left(\mathbf{x}_{k}-\mathbf{x}_{k}^{i}\right),
$$

where $\mathbf{p}_{\mathrm{A}, l: k}$ and $\mathbf{v}_{\mathrm{D}, l: k}$ are the HPR and DVL measurement sequence from time $l$ to $k$, respectively, and $\delta(\cdot)$ is the Dirac function. Following the estimated posterior density, the ROV kinematics can be obtained by taking the weighted sum of the empirical distribution as

$$
\hat{\mathbf{x}}_{k}=\sum_{i=1}^{N s} w_{k}^{i} \mathbf{x}_{k}^{i} \text {. }
$$

\section{FAILURE MODES ANALYSIS}

When sensors suffer from faults, their measurement models are different from the fault-free conditions. This section gives the measurement models in different failure modes. These models will be used in the fault tolerant navigation system to detect and handle the failures. In the following, the sensor failure modes are named as $\mathcal{M}$ with superscript representing the sensor and a number representing the failure mode index.

Failure mode $\mathcal{M}^{\mathrm{HPR}, 1}$ - Loss of HPR measurement The previous HPR measurement model in (5) is valid when the ROV is in shallow water, where the HPR holds a constant update rate. It is known that the HPR update rate depends on the slant range between the transponder and the transducer. So when the ROV dives down to deep water, the HPR update rate becomes lower and uneven. This phenomenon is observed in the sea trial and shown in Figure 2. The nonuniform update rate of the acoustic measurement must be accommodated.

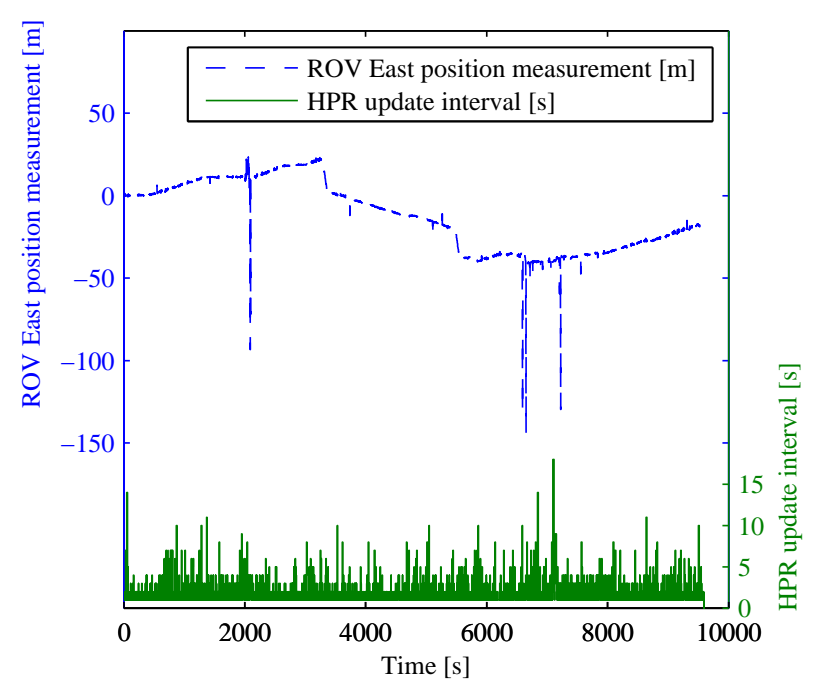

Fig. 2. A segment of the acoustic position measurement and its update interval. Notice that the horizontal axis is the measurement index instead of time.

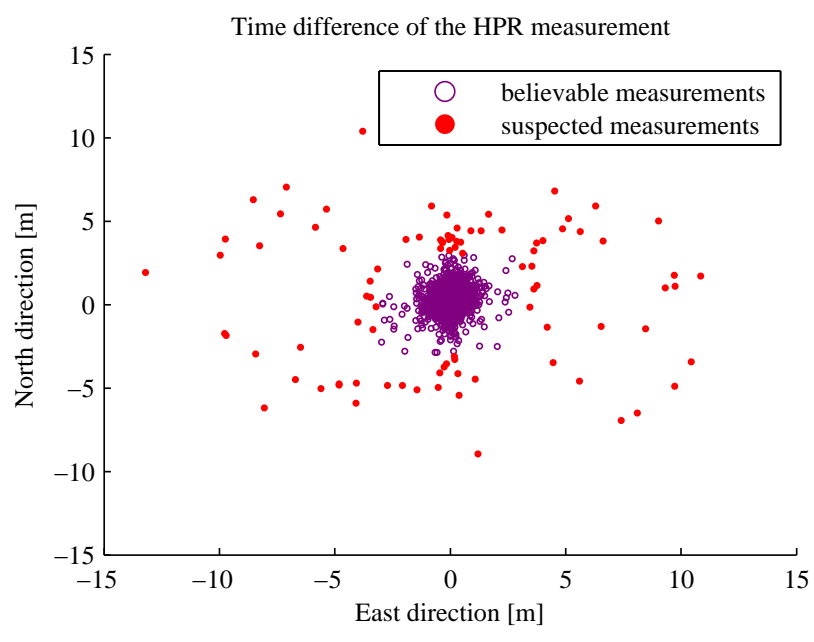

Fig. 3. The difference of the HPR measurement. Some extremely large difference, which are out of the range of this figure, are ignored.

Failure mode $\mathcal{M}^{\mathrm{HPR}, 2}$ - HPR measurement outliers Also shown in Figure 2, the position measurement frequently suffers from outliers. Figure 3 shows the time difference of the HPR measurements on a $2-\mathrm{D}$ plane. The difference is obtained by subtracting each measurement from the successor. Since the velocity of the ROV is low, measurements with large differences are suspected to be outliers. In Gustafsson (2001), the measurement outliers are seen as samples from another process whose measurement noise variance is significantly greater than the one in the normal case. That is, the outliers in the HPR measurement are subject to the distribution

$$
\mathbf{N}\left(\mathbf{C}_{\mathrm{p}} \mathbf{x}_{k}, \boldsymbol{\Sigma}_{\mathrm{A}, o}\right),
$$

where $\boldsymbol{\Sigma}_{\mathrm{A}, \mathrm{o}}$ is conceptually chosen as $\left\|\boldsymbol{\Sigma}_{\mathrm{A}, \mathrm{o}}\right\| \gg\left\|\boldsymbol{\Sigma}_{\mathrm{A}}\right\|$.

Failure mode $\mathcal{M}^{\mathrm{DVL}, 1}$ - Loss of DVL measurement When the DVL looses sea bottom tracking, it reports a sentinel max velocity, thus we lose the velocity measurement at this moment. 


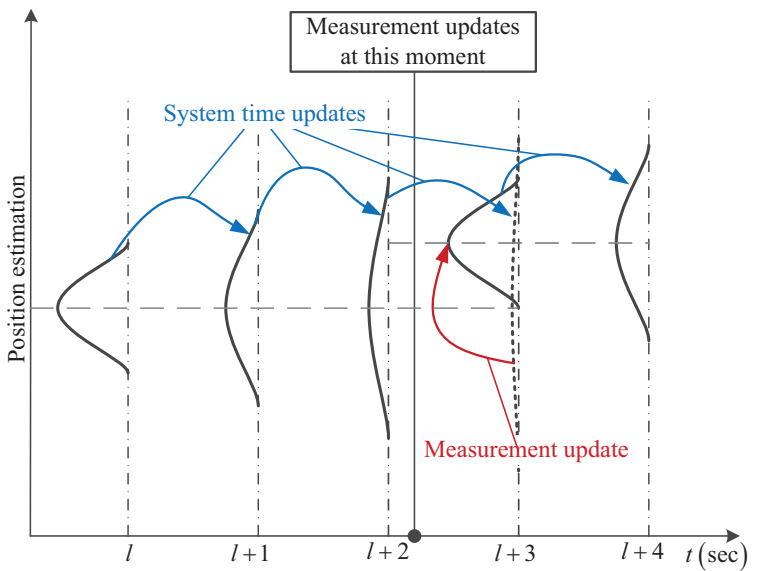

Fig. 4. Concept of the measurement synchronization for scalar system.

Simultaneous failures In the sea trial, it is also observed that the HPR and DVL failures can happen simultaneously, which results in the following two simultaneous failure modes.

(1) When $\mathcal{M}^{\mathrm{HPR}, 1}$ and $\mathcal{M}^{\mathrm{DVL}, 1}$ happen at the same time, the system loses all measurement.

(2) $\mathcal{M}^{\mathrm{HPR}, 2}$ and $\mathcal{M}^{\mathrm{DVL}, 1}$ happen at the same time; that is, the DVL measurement is lost and the HPR measurement is an outlier. This condition can be more harsh. A navigation system without a robust fault tolerant algorithm may believe the HPR reports a fault-free measurement, since there is no other measurement as a reference. Once the faulty measurement is involved in the estimation of the ROV position, the navigation system calculates an incorrect value, which is unlikely to be removed afterwards.

\section{PARTICLE FILTER BASED FAULT TOLERANT ROV NAVIGATION}

In order to accommodate the above mentioned failure modes, a particle filter based fault tolerant ROV navigation system is proposed in this section. This fault tolerant navigation algorithm supports asynchronous measurement update, system mode augmented system state, and switching between observation models.

\subsection{Asynchronous measurement update}

The navigation algorithm is desired to run in an asynchronous manner to accommodate the HPR and DVL measurement drop out $\mathcal{M}^{\mathrm{HPR}, 1}$ and $\mathcal{M}^{\mathrm{DVL}, 1}$. That is, the time update of the particle filter runs at every time step, while the measurement update only runs whenever a new measurement is obtained (Bar-Shalom et al. (2001)).

The particle filter runs its time update step in each time to continuously estimate the propagation of the distribution of the states over time by drawing new samples from (A.1). If failure mode $\mathcal{M}^{\mathrm{HPR}, 1}$ occurs, the particle filter skips the position measurement update, such that the weights of the particles will be updated according to

$$
w_{k}^{i} \propto w_{k-1}^{i} p\left(\mathbf{v}_{\mathrm{D}, k} \mid \mathbf{x}_{k}^{i}\right),
$$

where the conditional probability density function follows from equation (10). When the DVL measurement is lost
Table 1. System flag state transition probability.

\begin{tabular}{|r|c|c|c|c|}
\hline \multicolumn{2}{|c|}{$\mathrm{P}_{\mathrm{ij}}$} & \multicolumn{3}{|c|}{$\mathcal{M}^{\mathrm{HPR}, \mathrm{i}}$} \\
\cline { 3 - 5 } \multicolumn{2}{|c|}{$\mathcal{M}^{\mathrm{HPR}, 0}$} & $\mathcal{M}^{\mathrm{HPR}, 1}$ & $\mathcal{M}^{\mathrm{HPR}, 2}$ \\
\hline \multirow{3}{*}{$\mathcal{M}^{\mathrm{HPR}, \mathrm{j}}$} & $\mathcal{M}^{\mathrm{HPR}, 0}$ & 0.98 & 0.95 & 0.99 \\
\cline { 2 - 5 } & $\mathcal{M}^{\mathrm{HPR}, 1}$ & $\mathrm{~N} / \mathrm{A}$ & $\mathrm{N} / \mathrm{A}$ & $\mathrm{N} / \mathrm{A}$ \\
\cline { 2 - 5 } & $\mathcal{M}^{\mathrm{HPR}, 2}$ & 0.02 & 0.05 & 0.01 \\
\hline
\end{tabular}

as failure mode $\mathcal{M}^{\mathrm{DVL}, 1}$, the particle weights are updated by

$$
w_{k}^{i} \propto w_{k-1}^{i} p\left(\mathbf{p}_{\mathrm{A}, k} \mid \mathbf{x}_{k}^{i}\right)
$$

where the conditional probability density function follows (9). When the system is experiencing simultaneous failures $\mathcal{M}^{\mathrm{HPR}, 1}$ and $\mathcal{M}^{\mathrm{DVL}, 1}$, the particle filter skips the measurement update, such that the weights of the particle preserve the value at last time instance as

$$
w_{k}^{i}=w_{k-1}^{i} \text {. }
$$

Thus, by doing the measurement update asynchronously, the particle filter is able to tackle the measurement drop out failure modes.

\subsection{Switching observation models for outliers detection in the HPR measurements}

The outliers are defined as a sample from another distribution with large variance, as described by (13). While in fault-free condition, the measurements should be given by the distribution (5). Then the task of the outliers detection transforms into identifying from which distribution the measurements originate.

In order to embed the outliers detection into the particle filter, we augment the system model with a flag state $m \in\left\{\mathcal{M}^{\mathrm{HPR}, 0}, \mathcal{M}^{\mathrm{HPR}, 1}, \mathcal{M}^{\mathrm{HPR}, 2}\right\}$ representing the status of the HPR measurement, and $m_{k}$ is the flag state at time $k$. The notations $\mathcal{M}^{\mathrm{HPR}, 1}$ and $\mathcal{M}^{\mathrm{HPR}, 2}$ follows the corresponding failure modes in Section 4 , and $\mathcal{M}^{\mathrm{HPR}, 0}$ means the HPR measurement is fault-free. Then the detection problem of the HPR measurement is transformed into the estimation problem of sequence $m_{k}(k=1,2, \ldots)$. Assuming that the probability of outliers occurrence does not change with time, we can define a stationary Markov chain, such as

$$
\operatorname{Pr}\left(m_{k+1}=\mathcal{M}^{\mathrm{HPR}, \mathrm{j}} \mid m_{k}=\mathcal{M}^{\mathrm{HPR}, \mathrm{i}}\right)=P_{\mathrm{ij}},
$$

to describe the mode transition, where $i, j \in\{0,1,2\}$, and $P_{\mathrm{ij}}$ denotes the probability of the system flag state $m$ transfer from $m_{i}=\mathcal{M}^{\mathrm{HPR}, \mathrm{i}}$ to $m_{k+1}=\mathcal{M}^{\mathrm{HPR}, \mathrm{j}}$.

For instance, as shown in Table 1, if the measurement is fault-free, the probability of the next measurement is an outlier is $P_{02}=0.02$. As observed in the sea trial, if the HPR measurement is lost, the probability of "the next HPR measurement is an outlier" is higher and assigned as $P_{12}=0.05$. Also concluded from the experimental data, the probability that the two outliers come in sequence is low. Hence, we assign the prior $P_{22}=0.01$. Because the failure mode $\mathcal{M}^{\mathrm{HPR}, 1}$ can be decided deterministically whenever the HPR measurement is lost, the transition probabilities to mode $m_{k+1}=\mathcal{M}^{\mathrm{HPR}, 1}$ are not considered and denoted as "N/A" in Table 1. 


\subsection{Fault tolerant navigation algorithm}

The newly defined flag state $m$ and its transition equation (17) combining with the model (8)-(10) forms a new system model. The particle filter algorithm is modified according to the description in Appendix $\mathrm{B}$ in addition to the algorithm given in Appendix A to work extensively on this model.

The marginal probability mass of each system mode is calculated as

$$
\begin{aligned}
& \operatorname{Pr}\left(m_{k}=\mathcal{M}^{\mathrm{HPR}, m} \mid\left[\mathbf{p}_{\mathrm{A}, l: k} \mathbf{v}_{\mathrm{D}, l: k}\right]\right) \\
= & \sum_{i=1}^{N_{s}} w_{k}^{i} D\left(m_{k}^{i}, \mathcal{M}^{\mathrm{HPR}, m}\right)
\end{aligned}
$$

where the function $D(\cdot, \cdot)$ is defined as

$$
D(\xi, \zeta)=\left\{\begin{array}{ll}
1 & \xi=\zeta \\
0 & \xi \neq \zeta
\end{array} .\right.
$$

Then the estimated system mode $\hat{m}_{k}$ is obtained by picking up the mode with the largest marginal probability mass. The ROV kinematics is estimated by taking the expectation of the posterior density expanded by the particles with the estimated system mode $\hat{m}_{k}$, as

$$
\hat{\mathbf{x}}_{k}=\sum_{i=1}^{N s} w_{k}^{i} \mathbf{x}_{k}^{i} D\left(m_{k}^{i}, \hat{m}_{k}\right) .
$$

\section{SIMULATION}

The proposed fault tolerant navigation algorithm has been examined by simulation. The simulation uses the recorded sensor measurements from an ROV sea trial as input to the particle filter. However, some faults are added to the sensor measurements artificially to test the fault tolerant capability of the navigation algorithm. In the simulation, 1000 particles are used in the particle filter. At $5 \mathrm{~Hz}$ sampling frequency, the particle filter is able to processes 600 seconds real-time data within 340 seconds computing time on a standard laptop computer ${ }^{1}$ in the Matlab/Simulink environment. Hence, the computation load of the particle filter is acceptable.

In the simulation, the HPR measurement noise covariance matrix takes the value $\left[\begin{array}{lll}0.2111 & 0.0181 \\ 0.0181 & 0.0997\end{array}\right]$, which is calculated from the whole dataset with equation (4), and the DVL measurement noise covariance matrix is $\left[\begin{array}{cc}0.1 & 0 \\ 0 & 0.1\end{array}\right]$. The system mode transition probabilities are shown in Table 1.

A representative segment is picked out from the entire simulation result, which is shown in Figure 5, where (a) and (b) are the ROV position measurement and the estimated position in North and East direction, respectively; (c) and (d) show the ROV surge and sway speed measurement and estimation, respectively; and (e) shows the estimated system mode.

When the sensors are fault-free (Mode\#0), the proposed navigation algorithm accurately estimates the position and velocity of the ROV. The estimation error in position is generally small, and there is no significant delay in the velocity estimation.

1 The computer equipped 64 -bit dual core $\mathrm{CPU}$ at $2.93 \mathrm{GHz}$, but only one core is used in the simulation.
When the system mode is estimated between Mode\#1 to Mode\#5, the sensors are detected as faulty. Then the measurement update in the filter follows Table B.1.

During the period of an HPR drop out (from 470 sec. to 550 sec.), the navigation system estimates the ROV kinematic states only with velocity measurements, which is commonly referred to as dead reckoning. The estimated position at the end of this period is close to the HPR measured position. Around 525 sec., neither HPR nor DVL measurements are available, so that the system mode is determined as Mode\#4 and the weight update follows (16). Since the process noise of the particle filter is zero-mean Gaussian, the velocity estimation trends in short term to hold the last value before losing the DVL measurement. During the short term HPR dropout (about $220 \mathrm{sec}$ ), a smooth estimation of the position is obtained by dead reckoning, which improves the performance of the ROV control.

There are several HPR outliers in this segment. All of them are detected by the particle filter, with the result that these outliers have little effect on the estimation performance. Following the algorithm, the HPR outliers are detected since the velocity measurement does not support this significant position variation. Especially, there are outliers at about $305 \mathrm{sec}$. and $315 \mathrm{sec}$. when the DVL drops out. These measurements are detected as outliers since they are far beyond the expectation of the model.

During the period of DVL dropouts, the position of the ROV is estimated based only on the HPR measurements, while the ROV velocity is reconstructed from the position estimation.

In general, the results show that the state estimation performance is acceptable in the different conditions. In addition, outliers and dropouts of the sensors are handled well.

\section{CONCLUSION}

A particle filter based fault tolerant navigation algorithm for an ROV with HPR and DVL measurements was presented in this paper. To detect the sensor faults, a flag state representing the system mode was introduced, such that the modified particle filter algorithm could extensively run on this model. With this augmented model, the particle filter could estimate the system mode and the ROV kinematic states at the same time. A simulation study showed that the proposed navigation algorithm was able to detect the faults in the sensors, with the result of accurate and fault-free estimation of the ROV kinematic states.

The current research is to use the thrust commands of the ROV controller to complete the navigation model. A new experiment is also planned.

\section{ACKNOWLEDGEMENTS}

This work has been carried out at the Centre for Ships and Ocean Structures (CeSOS), and with the support from Applied Underwater Robotics Laboratory (AUR-Lab) at NTNU. Thanks also to the RCN research project 199567 , "Arctic DP", for funding the last author. 
Table B.1. Measurement update equations according to system mode

\begin{tabular}{cc|c}
\hline \hline \multicolumn{2}{c|}{ System mode } & Measurement update equations \\
\hline$\# 0$ & no fault & $(9)$ and $(10)$ \\
$\# 1$ & $\mathcal{M}^{\mathrm{HPR}, 1}$ & $(10)$ \\
$\# 2$ & $\mathcal{M}^{\mathrm{HPR}, 2}$ & $(13)$ and $(10)$ \\
\hline$\# 3$ & $\mathcal{M}^{\mathrm{DVL}, 1}$ & $(9)$ \\
$\# 4$ & $\mathcal{M}^{\mathrm{DVL}, 1}, \mathcal{M}^{\mathrm{HPR}, 1}$ & no measurement update, as $(16)$ \\
$\# 5$ & $\mathcal{M}^{\mathrm{DVL}, 1}, \mathcal{M}^{\mathrm{HPR}, 2}$ & $(13)$ \\
\hline \hline
\end{tabular}

Appendix A. PARTICLE FILTER ALGORITHM

(1) Initialising: Assume the particle filter starts to estimate the system states at time $l$. Then the particles are initialized with the sensor measurements at time $l-1$ as $\mathbf{x}_{l-1}^{i}=\left[\left(\mathbf{p}_{l-1}^{i}\right)^{\top}\left(\mathbf{v}_{l-1}^{i}\right)^{\top}\right]^{\top}\left(i=1, \cdots, N_{s}\right)$, where $\mathbf{p}_{l-1}^{i}$ are drawn from distribution (9) with $k=l-1$, and $\mathbf{v}_{l-1}^{i}$ are drawn from distribution (10) also with $k=l-1$, and $N_{s}$ is the number of particles in the particle filter. The weights of these particles are initialized as $w_{l-1}^{i}=1 / N_{s}$.

(2) Time update: The new particles are drawn from the importance density $q\left(\mathbf{x}_{k} \mid \mathbf{x}_{k-1}^{i},\left[\mathbf{p}_{\mathrm{A}, k} \mathbf{v}_{\mathrm{D}, k}\right]\right)=$ $p\left(\mathbf{x}_{k} \mid \mathbf{x}_{k-1}^{i}\right)$. That is, $\mathbf{x}_{k}^{i}$ are drawn from the distribution

$$
p\left(\mathbf{x}_{k} \mid \mathbf{x}_{k-1}^{i}\right)=\mathbf{N}\left(\mathbf{A}_{x} \mathbf{x}_{k-1}^{i}, \operatorname{Cov}\left(\mathbf{B}_{x} \mathbf{w}_{a, k}\right)\right) .
$$

(3) Measurement update: At this step, the sensor measurements are used to update the weights of the particles. Since the importance density has been chosen as (A.1), the update process of the weights follows

$$
\begin{aligned}
w_{k}^{i} & \propto w_{k-1}^{i} p\left(\left[\mathbf{p}_{\mathrm{A}, k} \mathbf{v}_{\mathrm{D}, k}\right] \mid \mathbf{x}_{k}^{i}\right) \\
& \propto w_{k-1}^{i} p\left(\mathbf{p}_{\mathrm{A}, k} \mid \mathbf{p}_{k}^{i}\right) p\left(\mathbf{v}_{\mathrm{D}, k} \mid \mathbf{v}_{k}^{i}\right) .
\end{aligned}
$$

(4) Re-sampling: All commonly recognized re-sampling methods are suitable for this PF algorithm. The PF in this research uses the residual re-sampling method proposed in Liu and Chen (1998) to prevent the degeneracy problem.

(5) Go to step 2 and start the next cycle.

\section{Appendix B. MODIFICATION OF PARTICLE FILTER ALGORITHM}

The particle filter running on the augmented ROV model with fault flag state $m$ is based on the basic particle filter algorithm given in Appendix A, and with following modifications.

(1) Initialising: The flag state of the particles are initialized as $m_{l-1}^{i}=\mathcal{M}^{\mathrm{HPR}, 0}$. This means we assume that the HPR measurement is fault-free at the beginning.

(2) Time update: For each particle, besides the time update of the vector $\mathbf{x}_{k}^{i}$, the flag state $m_{k}^{i}$ are drawn from the conditional probability mass function (17).
The exception is when the HPR measurement is not available, in which case all particles adopt flag state $\mathcal{M}^{\mathrm{HPR}, 1}$ deterministically.

(3) Measurement update: According to the failure modes of the sensors, the particles adopt different measurement updating equations, as shown in Table B.1, where "no fault" is also listed as a failure mode for convenience.

(4) Resampling: The same as the original particle filter.

(5) Go to step 2 and start the next cycle.

\section{REFERENCES}

Arulampalam, M., Maskell, S., Gordon, N., and Clapp, T. (2002). A tutorial on particle filters for online nonlinear/non-Gaussian Bayesian tracking. IEEE Transactions on Signal Processing, 50(2), 174-188.

Bar-Shalom, Y., Li, X.R., and Kirubarajan, T. (2001). Estimation with Applications to Tracking and Navigation: Theory Algorithms and Software.

Blain, M., Lemieux, S., and Houde, R. (2003). Implementation of a ROV navigation system using acoustic/Doppler sensors and Kalman filtering. In Proc. OCEANS 2003, volume 3, 1255-1260. IEEE.

Caron, F., Davy, M., Duflos, E., and Vanheeghe, P. (2007). Particle filtering for multisensor data fusion with switching observation models: application to land vehicle positioning. IEEE Transactions on Signal Processing, 55(6), 2703-2719. doi:10.1109/TSP.2007.893914.

Dukan, F., Ludvigsen, M., and S\{\o\}rensen, A. (2011). Dynamic positioning system for a small size ROV with experimental results. In $O C E A N S$, 2011, 1-10.

Gustafsson, F. (2001). Adaptive Filtering and Change Detection. John Wiley \& Sons, Ltd, Chichester, UK, 1 edition.

Kinsey, J., Eustice, R., and Whitcomb, L. (2006). A survey of underwater vehicle navigation: Recent advances and new challenges. In Proceedings of the 7 th Conference on Maneuvering and Control of Marine Craft (MCMC 2006). IFAC, Lisbon.

Kinsey, J. and Whitcomb, L.L. (2004). Preliminary field experience with the DVLNAV integrated navigation system for oceanographic submersibles. Control Engineering Practice, 12(12), 1541-1549.

Liu, J.S. and Chen, R. (1998). Sequential Monte Carlo Methods for Dynamic Systems. Journal of the American Statistical Association, 93(443), 1032-1044.

Tafazoli, S. and Sun, X. (2006). Hybrid System State Tracking and Fault Detection Using Particle Filters. IEEE Transactions on Control Systems Technology, 14(6), 1078-1087.

Yardim, C., Michalopoulou, Z.H., and Gerstoft, P. (2011). An Overview of Sequential Bayesian Filtering in Ocean Acoustics. IEEE Journal of Oceanic Engineering, 36(1), $73-91$.

Zhao, B., Blanke, M., and Skjetne, R. (2012). Particle Filter ROV Navigation using Hydroacoustic Position and Speed Log Measurements. In the 2012 American Control Conference [Accepted]. 


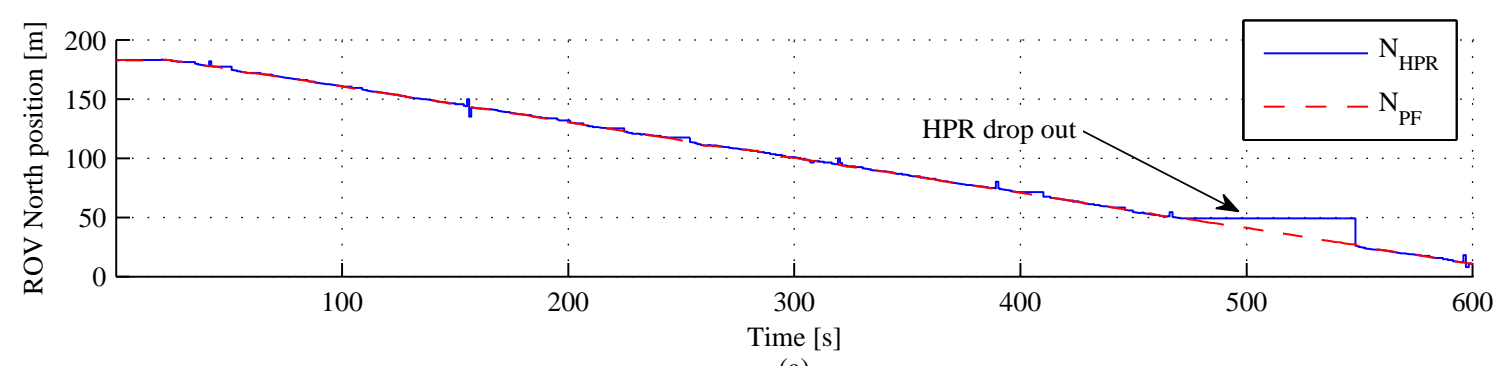

(a)

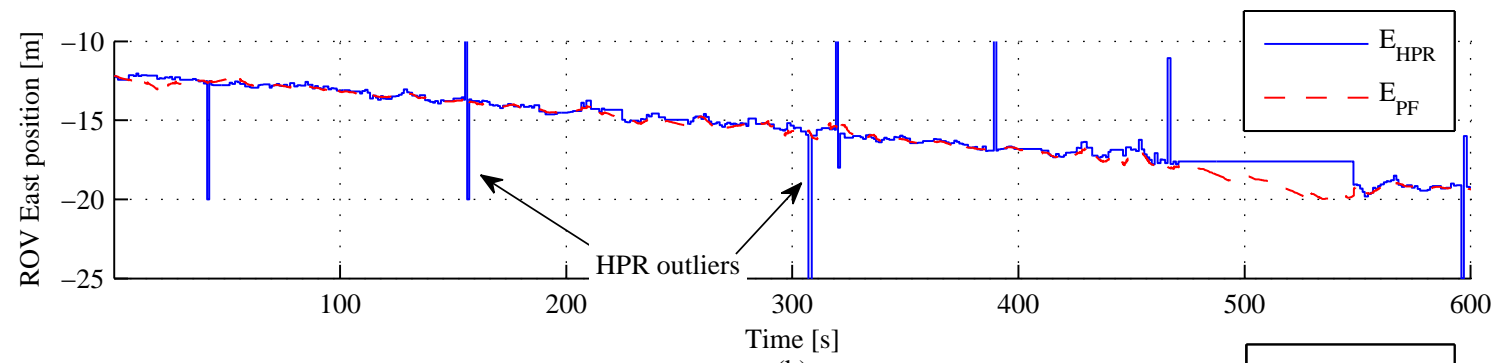

(b)

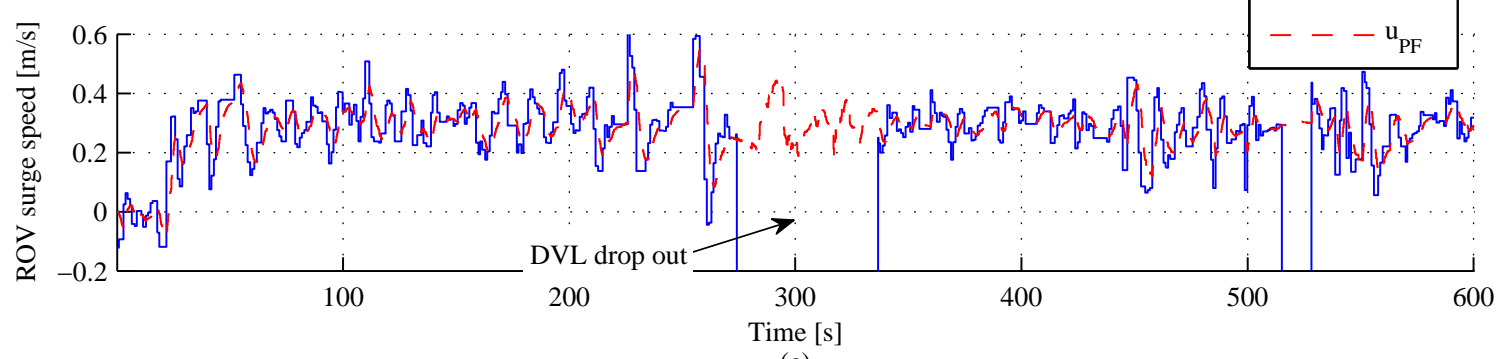

(c)

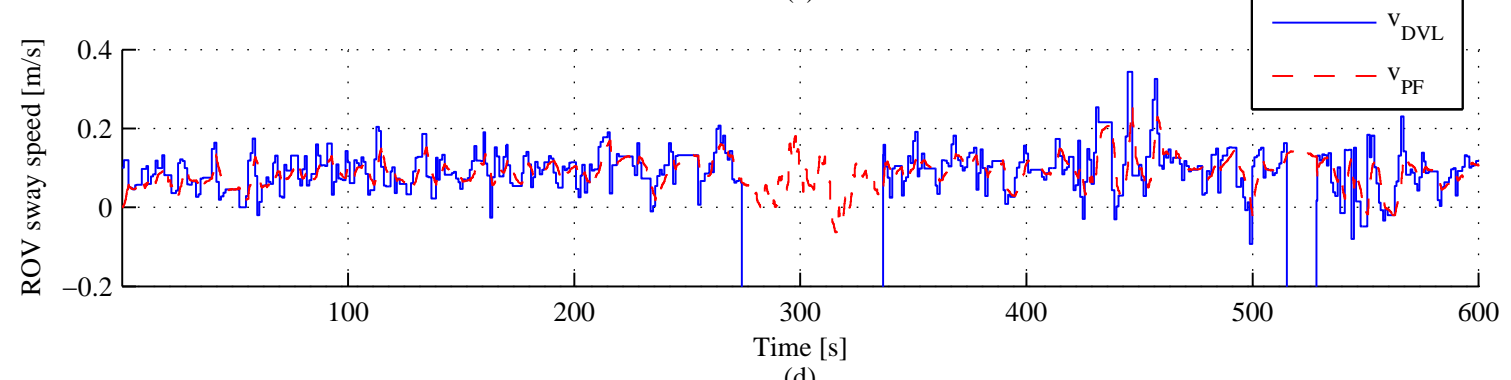

(d)

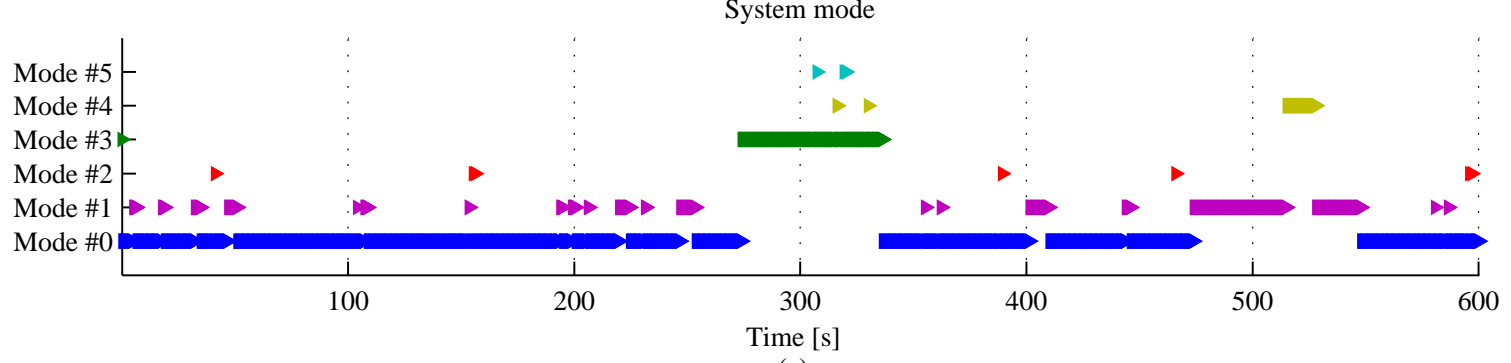

(e)

Fig. 5. Performance of the fault tolerant particle filter based ROV navigation. ROV position measurement and its estimation are in (a) and (b). ROV velocity and its estimation are in (c) and (d). The estimated system mode is in (e). Definitions of system modes in (e) refers to Table B.1. 